\title{
LEW, Rat Strain
}

National Cancer Institute

\section{Source}

National Cancer Institute. LEW, Rat Strain. NCI Thesaurus. Code C14407.

Of the large spectrum of 52 histologically different types of tumors, the highest incidence was observed for adenomas of the pituitary and adenomas/adenocarcinomas

of the adrenal cortex in both sexes, mammary gland tumors and endometrial carcinomas (45\%) in females, and C-cell adenomas/adenocarcinomas of the thyroid gland and tumors of the hemopoietic system (28\%) in males (Baum et al, 1995). 